\title{
Theoretical Analysis of Mining Induced Overburden Subsidence Boundary with the Horizontal Coal Seam Mining
}

\author{
Weitao Yan $\left(\mathbb{D},{ }^{1,2}\right.$ Junjie Chen $\mathbb{D}^{2},{ }^{2}$ Yi Tan $\mathbb{D}^{2},{ }^{2}$ Wenzhi Zhang, ${ }^{1}$ and Lailiang Cai ${ }^{1}$ \\ ${ }^{1}$ Henan Key Laboratory for Green and Efficient Mining \& Comprehensive Utilization of Mineral Resources, \\ Henan Polytechnic University, Jiaozuo, Henan 454003, China \\ ${ }^{2}$ State Collaborative Innovation Center of Coal Work Safety and Clean-efficiency Utilization, Jiaozuo, Henan 454003, China \\ Correspondence should be addressed to Junjie Chen; chenjj@hpu.edu.cn and Yi Tan; tanyi@hpu.edu.cn
}

Received 3 December 2020; Revised 4 February 2021; Accepted 1 March 2021; Published 30 March 2021

Academic Editor: Zhao-hui Chong

Copyright (C) 2021 Weitao Yan et al. This is an open access article distributed under the Creative Commons Attribution License, which permits unrestricted use, distribution, and reproduction in any medium, provided the original work is properly cited.

\begin{abstract}
The overburden subsidence induced by underground mining has caused great damage to the ecological environmental and seriously threatens the safe use of underground structures. Focusing on the overburden subsidence, this paper uses theoretical analysis method to study the overburden subsidence boundary with a horizontal coal seam mining. In this paper, the viscoelastic theory and the random medium theory are used separately to deduce and analyze the subsidence boundary of bedrock and unconsolidated stratum, which are two media with different lithology. For bedrock, the results show that the subsidence boundary of bedrock is $1 / 4$ of the wavelength of pressure wave from the mining boundary, strata subsidence boundary expands with the increase of vertical distance between calculated strata and coal seam, and the subsidence boundary in bedrock is an upward concave curve. For unconsolidated stratum, the results show that the larger the internal friction angle, the greater the angle between subsidence boundary and horizontal line. From the bottom to the surface of the unconsolidated stratum, the internal friction angle decreases gradually and the angle between subsidence boundary and horizontal line also decreases gradually, so the subsidence boundary curve in the unconsolidated stratum is convex. Combined with the bedrock and unconsolidated stratum, it is concluded that the subsidence boundary of the whole overburden is bowl-shaped. This study is helpful to reveal the black box of rock subsidence and can provide theoretical support for the establishment of overburden subsidence prediction model and transparent mine in the later stage.
\end{abstract}

\section{Introduction}

After mining of underground coal resources, the initial stress in rock mass is destroyed and redistributed, which leads to the large-scale subsidence of overburden and ground surface [1-5], and seriously affects the underground pipelines, structures, water system, ecological environment, and so on. Surface subsidence is only a representation, and overburden movement is the essence. Only by understanding the movement law of overburden, can we have a clear understanding of the subsidence fundamentally. The large-scale overburden movement results in the decline of groundwater table, reduction of farmland quality, destruction of ecological environment, decline of people's living standards, etc. [6-12]. In order to mitigate those adverse effects caused by mining subsidence, researchers have carried out a lot of work in the extraction method of subsidence information [13-15], analysis of subsidence law [16-18], revealing of subsidence mechanism [12, 19-21], establishment of subsidence prediction model [22-24], and development of mining methods with low ecological damage $[25,26]$. Taking subsidence prediction as an example, scholars have proposed some subsidence prediction method, such as empirical formula method, influence function method, section function method, chart method, and other surface subsidence prediction methods [22-24, 27-30]. The content of subsidence prediction includes two aspects: the degree of subsidence and the scope of subsidence. For the prediction of surface subsidence, no matter the degree or scope of subsidence, the current research is relatively sufficient. For 
the degree of subsidence of overlying strata, the prediction accuracy is relatively high. However, for the scope of subsidence of overlying strata, the study on boundary morphology of overburden subsidence is still insufficient. Therefore, it is necessary to study the subsidence boundary shape of overburden rock.

In this study, we consider bedrock and loose layer as viscoelastic medium and random medium, respectively. The theoretical analysis method was used to derive the shape of the subsidence boundary of overburden rock. Relevant research can provide knowledge support for further study of the overburden subsidence prediction methods.

\section{Traditional Mining Subsidence Model}

After the underground coal resources are mined out, the goaf is formed and the overburden and surface subsidence occur. When the size of the goaf is large enough, basin-like subsidence basin is formed on the surface, whereas bowl-like subsidence basin is formed on the surface. The boundary of surface subsidence basin generally refers to the outermost area of surface subsidence. According to the mining subsidence theory, because of the limitation of measuring instruments and methods, the point with subsidence of $10 \mathrm{~mm}$ was usually selected as the subsidence basin boundary. To define the subsidence boundary, the concept of angle of boundary was proposed in China and angle of draw was proposed in other countries, as shown in Figure 1.

(i) Angle of boundary: when the gob reached the critical size, or nearly so, on the major section of a movement basin, the angle between the horizontal line and the line connecting the surface subsidence boundary and the gob edge at the pillar side is the angle of boundary

(ii) Angle of draw: when the gob reached the critical size, or nearly so, on the major section of a movement basin, the angle between the vertical line and the line connecting the surface subsidence boundary and the panel edge at the pillar side is the angle of draw

From the above definition, it can be seen that they are mutually complementary, and they have the same effect. Therefore, we are just going to analyze the angle of draw.

For the angle of draw, the larger the angle is, the larger the influence range of subsidence is. According to the theory of mining subsidence, lithology is the main factor affecting the boundary angle. The harder the strata are, the smaller the angle is and the smaller the range of strata movement is. On the contrary, the softer the lithology is, the larger the angle is and the larger the range of strata movement is.

The above two definitions assume that the movement boundary in overlying strata simply conform to the linear law. However, many experimental results show that the movement boundary in overburden does not simply conform to the linear rule. Therefore, it is necessary for us to study the boundary shape in overburden strata.

\section{Theoretical Analysis}

3.1. Movement Boundary Shape of Bedrock. Based on the research results of Yan (2019), the bedrock are in line with the Kelvin rheological model.

Figure 2 shows the coordinate system which is suitable for the analysis of skewed subsidence characteristics of the rock mass. Establishing the $x$-axis along the central axis of rock beam, the part above the pillar is positive and the part above the gob is negative. Establish the $z$-axis in the vertical direction, and take the downward direction as positive. The coordinate origin point $\mathrm{O}$ is selected just above the gob boundary. Correspondingly, the ground coordinate system $\mathrm{XO}_{1} \mathrm{~W}$ has been established on the Earth's surface.

According to the results of Yan (2019), we can derive the deflection equation of the beam:

$$
\begin{aligned}
& W_{1}(x, t)=\frac{M P_{z}}{E_{k}} \frac{L_{p}^{2}}{L_{k}^{2}}\left(1-e^{-k t}\right) e^{-\left(\pi / L_{p}\right) x}\left[-\frac{L_{k}-L_{p}}{L_{k}+L_{p}} \sin \left(\frac{\pi}{L_{p}} x\right)+\cos \left(\frac{\pi}{L_{p}} x\right)\right]+\frac{M P_{z}}{E_{p}}, \quad x>0, \\
& W_{2}(x, t)=\frac{M P_{z}}{E_{k}}\left(1-e^{-k t}\right)\left\{e^{-\left(\pi / L_{k}\right) x}\left[\frac{L_{k}-L_{p}}{L_{k}+L_{p}} \sin \left(\frac{\pi}{L_{k}} x\right)-\cos \left(\frac{\pi}{L_{k}} x\right)\right]+1+\frac{L_{p}^{2}}{L_{k}^{2}}\right\}+\frac{M P_{z}}{E_{p}}, \quad x<0, \\
& L_{k}=L_{p} .
\end{aligned}
$$

where $L_{P}$ and $L_{k}$ are the half wavelength of pressure wave of the coal pillar side and the gob side, respectively, $M$ is the mining thickness, $P_{z}$ is the initial stress, $P_{z}=\gamma H$, and $E_{k}$ and $E_{P}$ are the elastic modulus of the goaf side and coal pillar side, respectively.

Because $M P_{z} / E_{p}$ is caused by the self-weight stress of overburden before mining and is not induced by mining, this item can be neglected. In addition, in order to facilitate calculation, we generally make the following approximate assumptions:
When the time tends to be infinite, the maximum subsidence value appears on the surface at a large distance from the coal pillar above the goaf:

$$
\lim _{\substack{t \longrightarrow \infty \\ x \longrightarrow-\infty}} W(x, t)=\frac{2 M P_{z}}{E_{k}}=W_{\max }
$$

where $W_{\max }$ is the surface maximum subsidence value. 


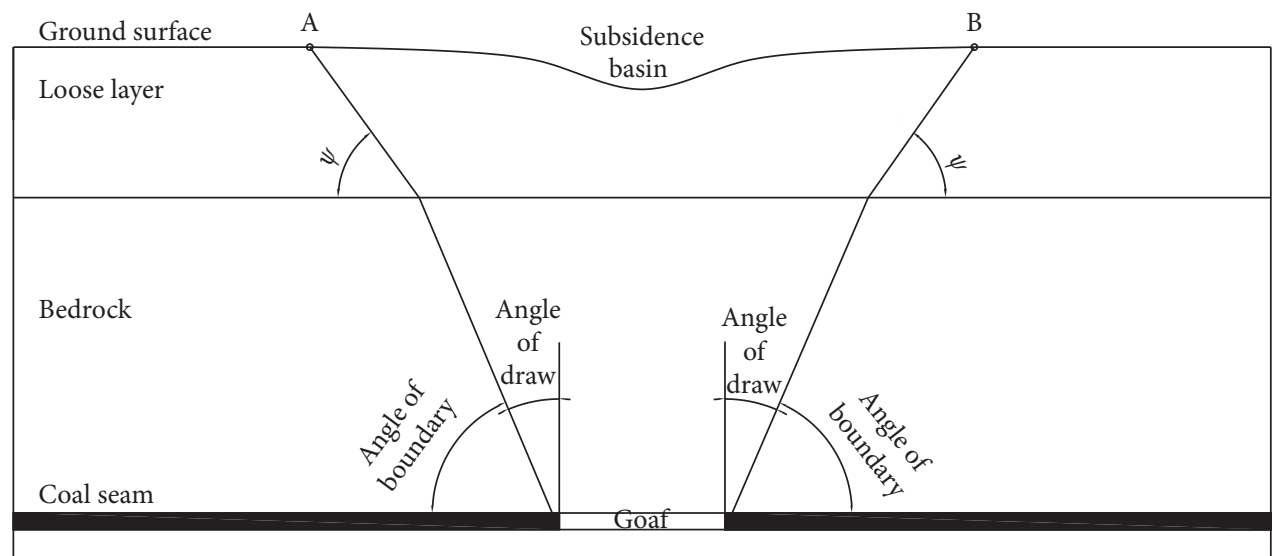

FIgURE 1: Definition of the inner boundary of overburden rock.

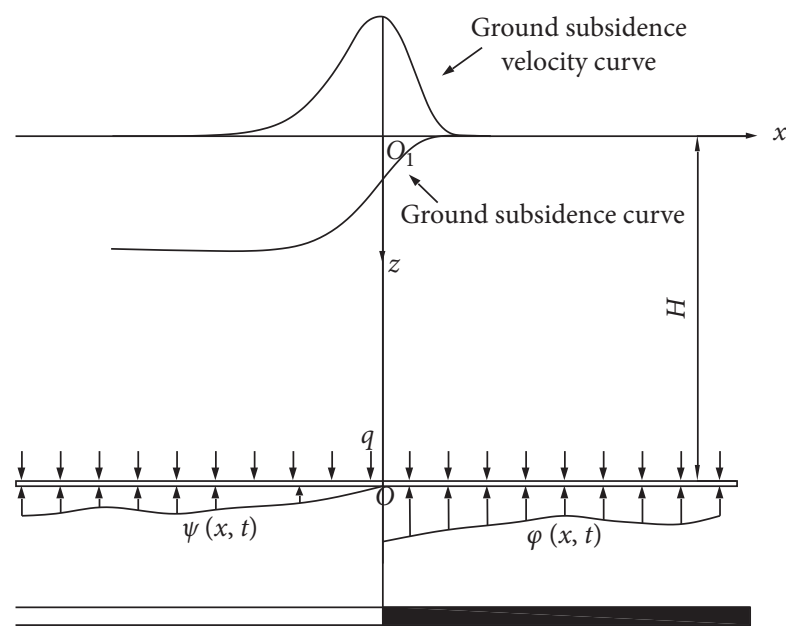

FIgURE 2: Construction of the coordinate system.

According to the above discussion, formula (1) can be simplified to

$$
\begin{aligned}
& W_{1}(x, t)=\frac{W_{\max }}{2}\left(1-e^{-k t}\right) e^{-(\pi / L) x} \cos \left(\frac{\pi}{L} x\right), \quad x>0, \\
& W_{2}(x, t)=\frac{W_{\max }}{2}\left(1-e^{-k t}\right)\left\{2-e^{(\pi / L) x} \cos (\pi / L) x\right\}, \quad x<0 .
\end{aligned}
$$

If the mining has gone through a long time and the time tends to be infinite, the above formula can be further simplified as follows:

$$
\begin{aligned}
& W_{1}(x)=\frac{W_{\text {max }}}{2} e^{-(\pi / L) x} \cos \left(\frac{\pi}{L} x\right), \quad x>0, \\
& W_{2}(x)=\frac{W_{\text {max }}}{2}\left\{2-e^{(\pi / L) x} \cos \left(\frac{\pi}{L} x\right)\right\}, \quad x<0 .
\end{aligned}
$$

Solving the equation $W_{1}(x)=0$, we can get $x=(L / 2)$.

Because $L$ represents half of the wavelength of pressure wave, the movement boundary of bedrock is $1 / 4$ of the wavelength of pressure wave from the mining boundary.
The rock beam of the coal pillar side is compressed in normal direction of strata and pulled in parallel direction. According to the theory of rock mechanics, the relationship between compressive stress and P-wave velocity is shown in Figure 3.

The relationship between compressive stress and P-wave velocity along the vertical direction of compressive stress can be obtained by fitting:

$$
v_{p}=(A-p)^{n}, n \in(0,1) .
$$

According to the general theory of coal mining subsidence, it can be seen that, in the vertical direction, the overburden subsidence of the coal pillar side and goaf side presents different changing rules:

(1) Coal pillar side: with a certain horizontal distance from the mining boundary, the farther the vertical distance from the mining seam is, the bigger the subsidence value is, and the logarithmic growth occurs

(2) Goaf side: with a certain horizontal distance from the mining boundary, the farther the vertical distance from the mining seam is, the smaller the subsidence value is, and it decreases logarithmically

The subsidence value of overburden rock on the coal pillar side is smaller than that on the goaf side in the same stratum.

According to the above rules, the following formulas can be used for calculating the subsidence value of overburden rock on the coal pillar side:

$$
w(z)=\ln (k z)
$$

where $z$ is the vertical distance from the mining seam.

According to [3, 4], the supporting reaction of underlying rock mass to the rock beam is proportional to the subsidence value of rock beam. It can be described as

$$
\varphi(z)=c \ln (k z), \quad(c>0) .
$$

The compressive stress of rock strata in vertical direction is equal to the overlying gravity minus the supporting reaction: 


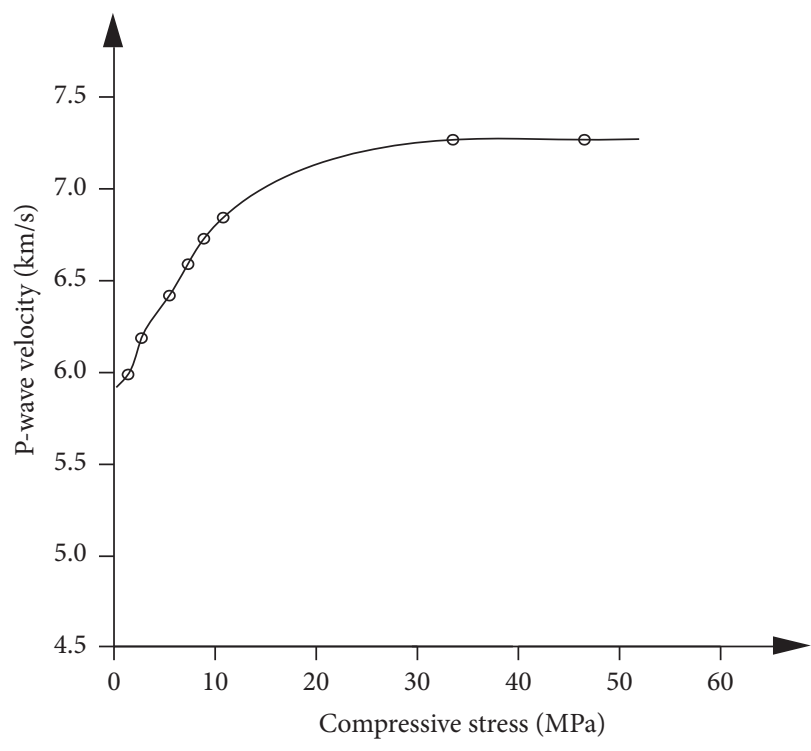

(a)

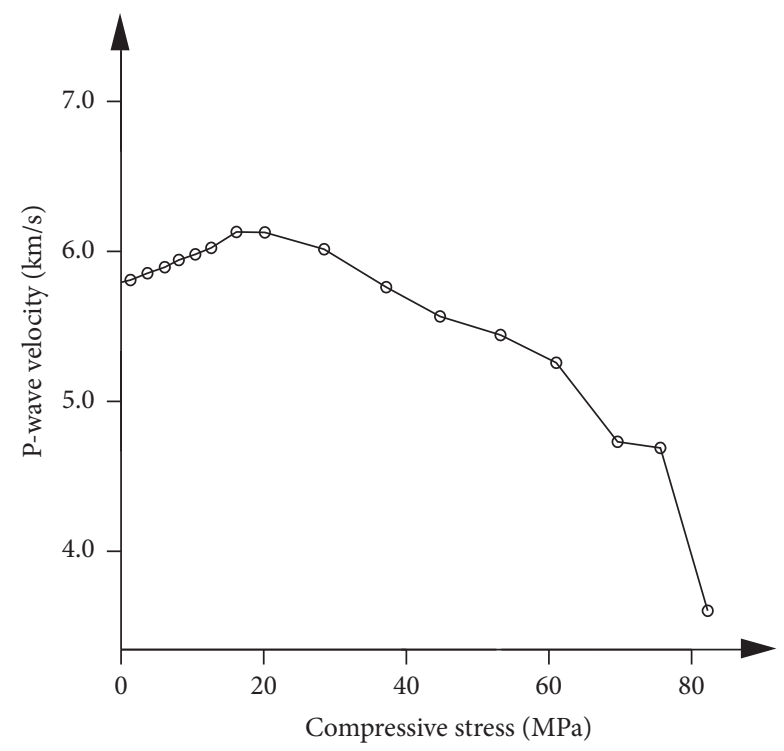

(b)

FIgUre 3: Variation curve of pressure wave velocity. (a) P-wave velocity along the direction of compressive stress. (b) P-wave velocity perpendicular to the direction of compressive stress.

$$
p=P_{z}-\varphi(\mathrm{z})=\gamma(H-z)-c \ln (k z)
$$

where $p$ is compressive stress, $P_{z}$ is overlying gravity, $\varphi$ is the supporting reaction, and $\gamma$ is bulk density.

Bringing formula (9) into (6), the relationship between $\mathrm{P}$-wave velocity perpendicular to the direction of compressive stress and the distance from the mining coal seam can be obtained:

$$
v_{p}=(A-\gamma H+\gamma z+c \ln (k z))^{n} .
$$

According to the wave theory, we know that wavelength equals wave velocity divided by frequency. So, the relationship between the $\mathrm{P}$-wave wavelength perpendicular to the compressive stress direction and the distance from it to the mining coal seam can be calculated by the following formula:

$$
L=\frac{v_{p}}{f}=\frac{A-\gamma H+\gamma z+c \ln (k z)^{n}}{f} .
$$

The relationship between the two can be illustrated by Figure 4.

It can be seen from the above that the movement boundary of strata expands with the increase of the vertical distance between the calculated strata and the mining coal seam, and the movement boundary of bedrock is concave as a whole (Figure 5).

3.2. Movement Boundary Shape of Loose Layer. Loose layer includes Quaternary and Neogene strata. It is composed of soil, sand, gravel, pebble layer, and so on. Compared with bedrock, the cohesive force between particles of the loose layer is small, and the strength of loose layer is very weak. From the knowledge of soil mechanics, we can see that the loose layer is similar to the random medium. Therefore, when analyzing the movement boundary of the loose layer, the loose layer can be generalized as the random medium. The analysis model is as follows.

As shown in Figure 6, when the random particle A moves away and the random particle $C$ slips toward its position. The force borne by the random particle $\mathrm{C}$ mainly consists of two parts: its own gravity Wand the horizontal thrust $T$ of the random particle B to it. Assuming that the angle between the movement boundary line and the horizontal plane is $\alpha$, the sliding force of random particle $\mathrm{C}$ along the movement boundary line is $\mathrm{R}_{f}=\mathrm{W} \sin \alpha$, and the normal stress perpendicular to the movement boundary is $N=\mathrm{W} \cos \alpha+T \sin \alpha$. The antisliding force generated by the normal stress is as follows:

$$
N \tan \varphi=(\mathrm{W} \cos \alpha+T \sin \alpha) \tan \varphi .
$$

The total antisliding force is as follows:

$$
R_{o}=(\mathrm{W} \cos \alpha+T \sin \alpha) \tan \varphi+T \cos \alpha \text {. }
$$

When $\mathrm{R}_{f}=R_{o}$, the random particle $\mathrm{C}$ is in the critical sliding state. The following formula can be obtained:

$$
\frac{\mathrm{W} \sin \alpha}{(\mathrm{W} \cos \alpha+T \sin \alpha) \tan \varphi+T \cos \alpha}=1 \text {. }
$$

After the above formula is processed, the following formula can be obtained:

$$
\tan \varphi=\frac{\mathrm{W}-T \cot \alpha}{T+\mathrm{W} \cot \alpha}
$$

According to the knowledge of soil mechanics, the deeper the loose layer is buried, the greater the compaction degree and the relative density are and the larger the internal 


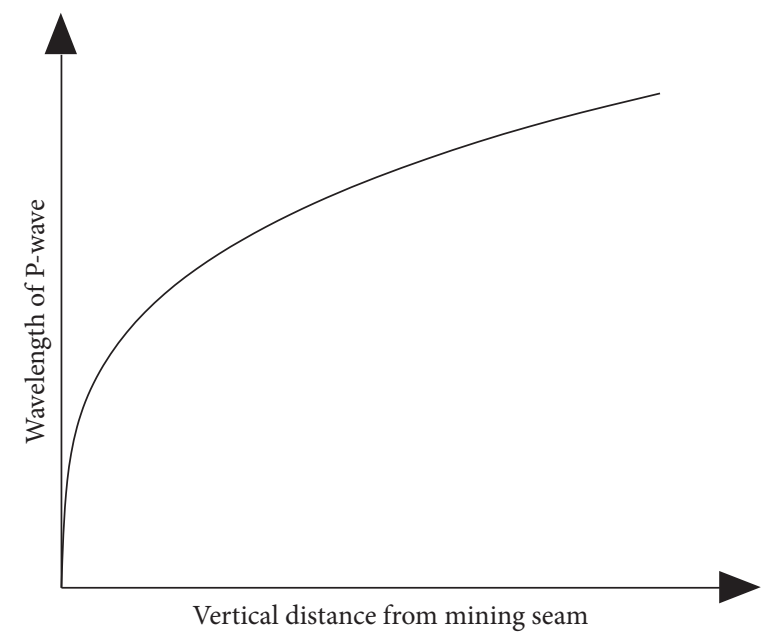

Figure 4: The relationship between the longitudinal wave length and the distance from it to the coal seam.

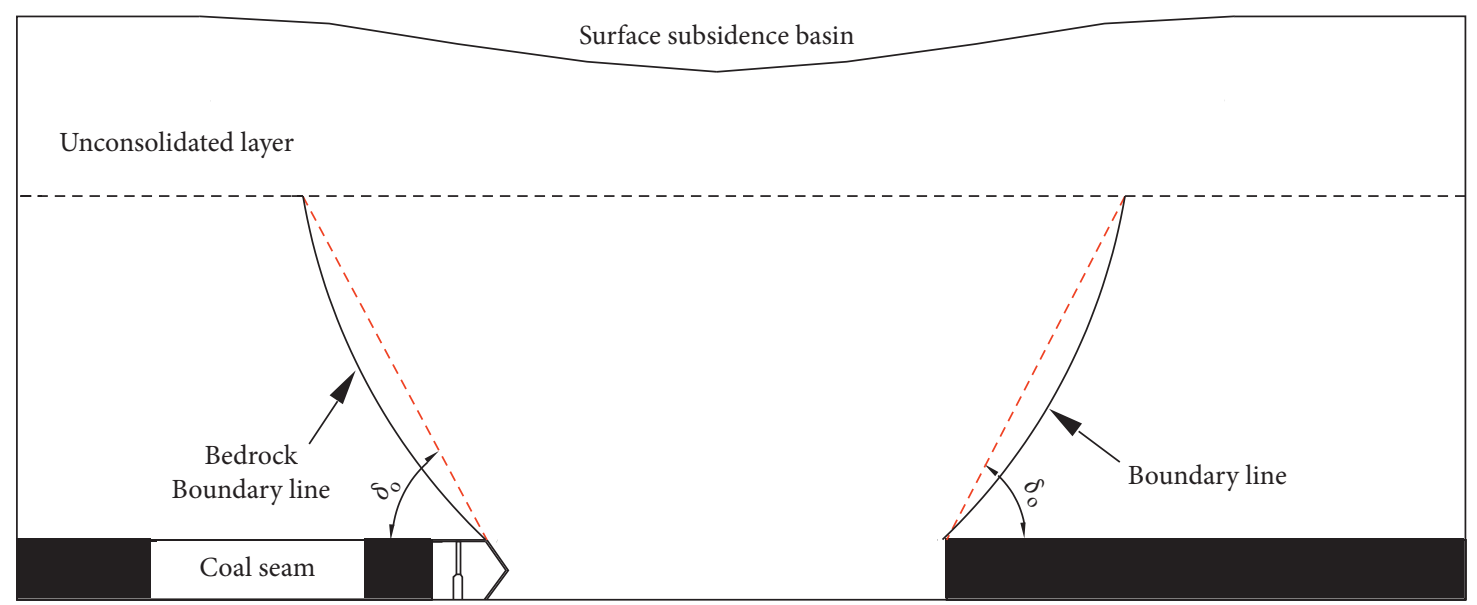

FIGURE 5: Movement boundary shape of bedrock.

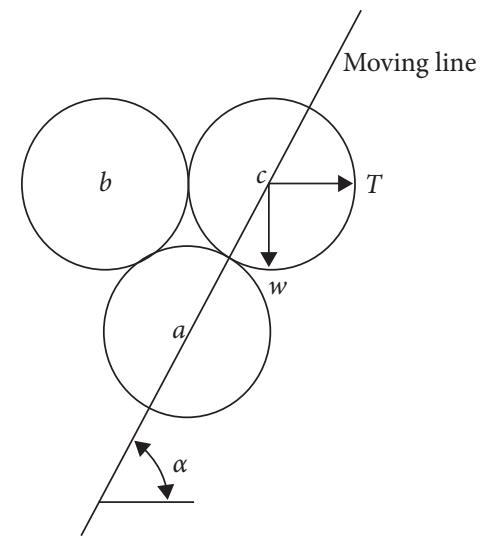

FIGURE 6: Analysis diagram of movement boundary of the loose layers.

friction angle is. Therefore, it can be concluded that, from the bottom to the surface of the loose layer, $\alpha$ gradually decreases, and the movement boundary line in the loose layer is convex. The shape of movement boundary is shown in Figure 7.

3.3. Movement Boundary Shape of Overlying Strata. According to the above discussion, the shape of movement boundary in bedrock is convex, while that in the loose layer is convex. The combination of the two is shown in Figure 8. It can be seen from the figure that the shape of the movement boundary in the whole overburden is bowl-shaped.

3.4. Application Scope. This movement boundary shape of overlying strata is a theoretical analysis model. As such, the application is limited. When the movement of the strata above the gob is only affected by self-weight and overburden pressure, the movement boundary shape is high. However, when a place has a wide range of geological structure (e.g., faults and folds) or geological events (e.g., earthquakes), the movement boundary shape model is unsuitable. 


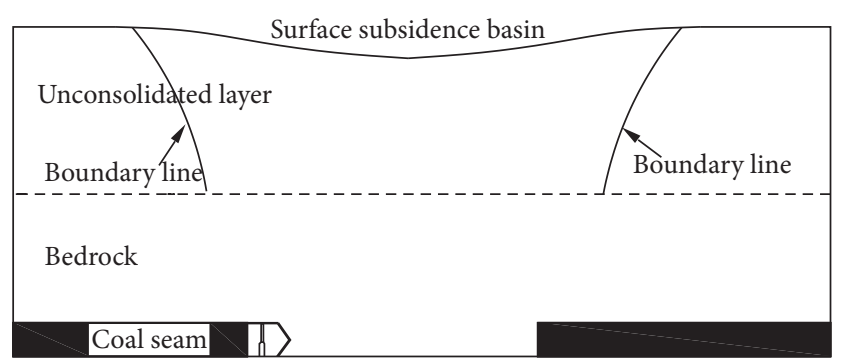

Figure 7: Movement boundary shape of the loose layer.

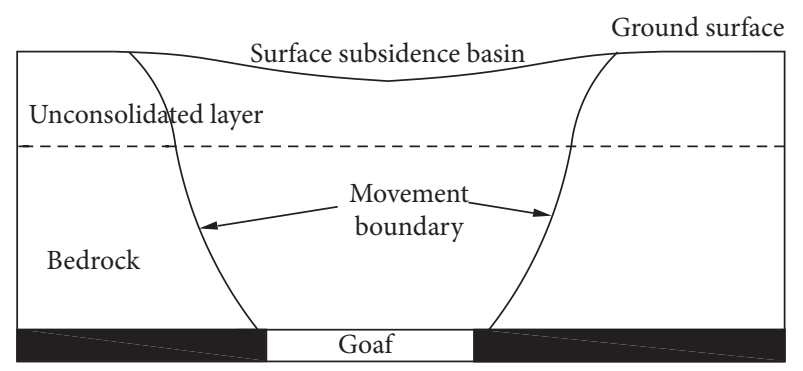

Figure 8: Movement boundary shape of overburden rock.

\section{Conclusions}

Based on the theoretical analysis, we have drawn the following conclusions.

(1) Loose layer and bedrock constitute overlying strata together, but the lithologies of these two materials are very different. In the process of boundary morphology analysis, these two materials were treated separately. The loose layer is regarded as random medium, and the bedrock is regarded as viscoelastic medium.

(2) The movement boundary of bedrock is $1 / 4$ th of the wavelength of pressure wave from the mining boundary, and the strata subsidence boundary expands with the increase of vertical distance between calculated strata and coal seam.

(3) Based on the elastic wave theory and random medium theory, it is deduced that the shape of movement boundary in the loose layer is convex, and the shape of movement boundary in bedrock is upper concave. The shape of the movement boundary of the whole overlying strata is similar to that of a bowl.

(4) Research results can provide theoretical support for the subsequent establishment of overlying rock subsidence prediction model and provide technical support for the protection of underground structures from the mining impacts.

\section{Data Availability}

The data used to support the findings of this study are available from the corresponding author upon request.

\section{Conflicts of Interest}

The authors declare no conflicts of interest.

\section{Acknowledgments}

This research was supported by the National Natural Science Foundation of China (Grant nos. U1810203 and 51974105), Henan Scientific and Technological Projection (Grant nos. 212102310012, 212102310414, and 202102310294), Key Scientific Research Projects of Higher Education Institutions in Henan Provience (Grant no. 20A440005), Research Fund of Henan Key Laboratory for Green and Efficient Mining and Comprehensive Utilization of Mineral Resources (Henan Polytechnic University) (Grant no. KCF202004), Open Fund of State Key Laboratory of Water Resource Protection and Utilization in Coal Mining (Grant nos. WPUKFJJ2019-20 and WPUKFJJ2019-17), Fundamental Research Funds for the Universities of Henan Province (Grant no. NSFRF200314), Youth Backbone Teacher Support Program of Henan Polytechnic University (Grant no. 2019XQG-07), and Doctoral Fund Program of Henan Polytechnic University (Grant no. B2017-07). All those financial supports are gratefully acknowledged.

\section{References}

[1] C. Chen and Z. Q. Hu, "Research advances in formation mechanism of ground crack due to coal mining subsidence in China," Journal of China Coal Society, vol. 43, no. 3, pp. 810-823, 2018.

[2] X. Cui, Y. Gao, and D. Yuan, "Sudden surface collapse disasters caused by shallow partial mining in Datong coalfield, China," Natural Hazards, vol. 74, no. 2, pp. 911-929, 2014.

[3] G. W. Fan, D. S. Zhang, and L. Q. Ma, "Overburden movement and fracture distribution induced by longwall mining of the shallow coal seam in the Shendong coalfield," Journal of China University of Mining \&Technology, vol. 40, no. 2, pp. 196-201, 2011.

[4] X. Yang, G. Wen, L. Dai, H. Sun, and X. Li, "Ground subsidence and surface cracks evolution from shallow-buried close-distance multi-seam mining: a case study in bulianta coal mine," Rock Mechanics and Rock Engineering, vol. 52, no. 8, pp. 2835-2852, 2019.

[5] X. Lian, H. Hu, H. Li, and D. Hu, "Main geological and mining factors affecting ground cracks induced by underground coal mining in Shanxi Province, China," International Journal of Coal Science \& Technology, vol. 7, no. 2, pp. 362-370, 2020.

[6] F. G. Bell, T. R. Stacey, and D. D. Genske, "Mining subsidence and its effect on the environment: some differing examples," Environmental Geology, vol. 40, no. 1-2, pp. 135-152, 2000.

[7] L. M. Fan, X. D. Ma, and R. J. Ji, "The progress of research engineering practice of water-preserved coal mining in western eco-environment frangible area," Journal of China Coal Society, vol. 40, no. 8, pp. 1711-1717, 2015.

[8] S. Sinha, R. N. Bhattacharya, and R. Banerjee, "Surface iron ore mining in eastern India and local level sustainability," Resources Policy, vol. 32, no. 1-2, pp. 57-68, 2007.

[9] S. Saha, S. K. Pattanayak, E. O. Sills, and A. K. Singha, "Undermining health: environmental justice and mining in India," Health \& Place, vol. 17, no. 1, pp. 140-148, 2011. 
[10] T. Sasaoka, H. Takamoto, T. Shimada, J. Oya, A. Hamanaka, and K. Matsui, "Surface subsidence due to underground mining operation under weak geological condition in Indonesia," Journal of Rock Mechanics and Geotechnical Engineering, vol. 7, no. 3, pp. 337-344, 2015.

[11] Z. Jing, J. Wang, Y. Zhu, and Y. Feng, "Effects of land subsidence resulted from coal mining on soil nutrient distributions in a loess area of China," Journal of Cleaner Production, vol. 177, pp. 350-361, 2018.

[12] J. Z. Wang, J. R. Kang, and Z. Q. Chang, "The mechanism analysis on the dissymmetry of the surface subsidence basin," Journal of China Coal Society, vol. 24, no. 3, pp. 252-255, 1999.

[13] H. D. Fan, L. Wang, and B. F. Wen etal, "A new model for three-dimensional Deformation extraction with single-track InSAR based on mining subsidence characteristics," International Journal of Applied Earth Observations and Geoinformation, vol. 94, Article ID 102223, 2021.

[14] M. Mohammady, H. R. Pourghasemi, and M. Amiri, "Spatial modeling of susceptibility to subsidence using machine learning techniques," Stochastic Environmental Research and Risk Assessment, 2021.

[15] C. Jiang, L. Wang, X. Yu, S. Chi, T. Wei, and X. Wang, "DPIM-based InSAR phase unwrapping model and a 3D mining-induced surface deformation extracting method: a case of Huainan mining area," KSCE Journal of Civil Engineering, vol. 25, no. 2, pp. 654-668, 2021.

[16] J. Ju and J. Xu, "Surface stepped subsidence related to top-coal caving longwall mining of extremely thick coal seam under shallow cover," International Journal of Rock Mechanics and Mining Sciences, vol. 78, pp. 27-35, 2015.

[17] Q. B. Guo, G. L. Guo, and J. F. Zha etal, "Research on the surface movement in a mountain mining area: a case study of Sujiagou mountain, China," Environmental Earth Sciences, vol. 75, Article ID 472, 2016.

[18] F.-Q. Tang, "Research on mechanism of mountain landslide due to underground mining," Journal of Coal Science and Engineering (China), vol. 15, no. 4, pp. 351-354, 2009.

[19] G. W. Cheng, T. H. Yang, H. Y. Liu et al., "Characteristics of stratum movement induced by downward longwall mining activities in middle-distance multi-seam," International Journal of Rock Mechanics and Mining Sciences, vol. 136, Article ID 104517, 2020.

[20] W. Yan, H. Dai, and J. Chen, "Surface crack and sand inrush disaster induced by high-strength mining: example from the Shendong coal field, China," Geosciences Journal, vol. 22, no. 2, pp. 347-357, 2018.

[21] Y. Sun, J. Zuo, S. Karakus, and J. Wang, "Investigation of movement and damage of integral overburden during shallow coal seam mining," International Journal of Rock Mechanics and Mining Sciences, vol. 117, pp. 63-75, 2019.

[22] W. T. Yan, J. J. Chen, and H. B. Chai, "Ground surface dynamic damage prediction model with high-strength mining in mining area," Transactions of the Chinese Society of Agricultural Engineering, vol. 35, no. 19, pp. 267-273, 2019.

[23] P. S. Ramesh and N. Y. Ram, "Prediction of subsidence due to coal mining in Raniganj coalfield, West Bengal, India," Engineering Geology, vol. 39, pp. 103-111, 1995.

[24] G. Ren, B. N. Whittaker, and D. J. Reddish, "Mining subsidence and displacement prediction using influence function methods for steep seams," Mining Science and Technology, vol. 8, no. 3, pp. 235-251, 1989.

[25] S. Chen, D. Yin, F. Cao, Y. Liu, and K. Ren, “An overview of integrated surface subsidence-reducing technology in mining areas of China," Natural Hazards, vol. 81, no. 2, pp. 1129-1145, 2016.

[26] G. L. Guo, H. Z. Li, and J. F. Zha, "An approach to protect cultivated land from subsidence and mitigate contamination from colliery gangue heaps," Process Safety and Environmental Protection, vol. 124, pp. 336-344, 2019.

[27] H. Li, J. Zha, and G. Guo, "A new dynamic prediction method for surface subsidence based on numerical model parameter sensitivity," Journal of Cleaner Production, vol. 233, pp. 1418-1424, 2019.

[28] Y. Luo and J. W. Cheng, "An influence function method based subsidence prediction program for longwall mining operations in inclined coal seams," Mining Science and Technology, vol. 19, pp. 0592-0598, 2009.

[29] H. Y. Dai and J. Z. Wang, "Prediction model for surface movement and deformation induced by sub-critical extraction," Journal of China Coal Society, vol. 28, no. 6, pp. 583587, 2003.

[30] W. Yan, J. Chen, and Y. Yan, “A new model for predicting surface mining subsidence: the improved lognormal function model," Geosciences Journal, vol. 23, no. 1, pp. 165-174, 2019. 